\title{
Managing obstructive gastric volvulus: challenges and solutions
}

\author{
Hector Alejandro \\ Rodriguez-Garcia' \\ Andrew S Wright ${ }^{2-4}$ \\ Robert B Yates ${ }^{1-3}$ \\ 'Department of Surgery, Center \\ for Esophageal and Gastric Surgery, \\ ${ }^{2}$ Center for Videoendoscopic \\ Surgery, ${ }^{3}$ Hernia Center, ${ }^{4}$ Institute \\ for Simulation and Interprofessional \\ Studies, UWMC, University of \\ Washington, Seattle, USA
}

This article was published in the following Dove Press journal:

Open Access Surgery

10 March 2017

Number of times this article has been viewed

Abstract: Gastric volvulus is the abnormal torsion of the stomach along its short or long axis. Most patients who experience gastric volvulus present with mild or intermittent gastric obstructive symptoms. However, severe acute gastric volvulus can result in complete gastric outlet obstruction and ischemia. Consequently, acute gastric volvulus warrants immediate evaluation and management. The goals of management are to relieve the obstruction and prevent recurrent volvulus. Techniques to manage gastric volvulus depend on patient characteristics and the presence of gastric ischemia. In the absence of gastric ischemia, gastric volvulus can be managed with anterior abdominal wall gastropexy or paraesophageal hernia repair. If gastric ischemia is present, operative resection of the affected portion of the stomach is indicated. When operative management is indicated, many patients with gastric volvulus can be managed with minimally invasive (laparoscopic, endoscopic, or laparoendoscopic) techniques.

Keywords: gastric volvulus, paraesophageal hernia, hiatal hernia

\section{Introduction}

Gastric volvulus is the abnormal torsion (>180 degrees) of the stomach. Classification of gastric volvulus can be made according to the specific anatomic abnormality, underlying etiology, and the time course of symptoms.

Organoaxial volvulus (approximately two-thirds of cases) occurs when the stomach rotates along its longitudinal axis (ie, a line connecting the gastric cardia and the gastric antrum) (Figure 1). Mesenteroaxial volvulus (approximately one-third of cases) occurs when the stomach rotates along its horizontal axis (ie, a line connecting the midpoint between the lesser curvature and the greater curvature of the stomach) (Figure 2).

The primary mechanism underlying gastric volvulus is the elongation and resulting laxity of the attachments of the stomach to its surrounding structures (eg, the diaphragm, spleen, liver, and colon). Although elongation of these structures may occur with the stomach in its normal subdiaphragmatic position, it is more commonly found that elongation of these structures - and subsequent gastric volvulus - occurs with a paraesophageal (PEH) or diaphragmatic (traumatic, Morgagni, Bochdalek) hernia. ${ }^{1-4}$

Some patients present with an incidental finding of gastric volvulus on a radiographic study obtained for another purpose. In these patients, the gastric volvulus is frequently incomplete ( $<180$ degrees) and therefore may not result in symptoms that the patient voluntarily reports. However, an astute physician who completes a detailed clinical history often will reveal longstanding heartburn and/or regurgitation, 


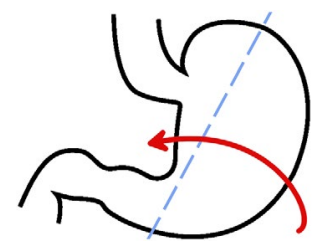

Figure I Organoaxial volvulus results from gastric torsion along the axis connecting the cardia and the antrum and results in obstruction at the cardia and/or the pylorus.

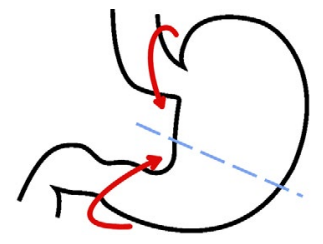

Figure 2 Mesenteroaxial volvulus results from gastric torsion along the axis connecting the lesser and greater curvatures.

Note: Obstruction occurs in the antropyloric zone due to cephalad torsion of the antrum (most common) or caudal torsion of the fundus (less common).

dysphagia, or early satiety and poor oral intake. For the remainder of patients who are symptomatic, gastric volvulus occurs either acutely or follows a more chronic, recurrent pattern.

For patients suffering from acute gastric volvulus, symptoms develop suddenly and include severe epigastric pain, nausea, and retching without productive emesis. These patients may present to the emergency department with severe and intractable symptoms. On the other hand, patients may present with chronic recurrent episodes of gastric volvulus that result in intermittent partial gastric outlet obstruction. In these cases, the symptoms are similar but less severe. As a result, patients may suffer from these episodes for months or years before they present for evaluation.

The management of gastric volvulus depends on the degree of gastric obstruction, the presence of gastric ischemia, and the presence of patient comorbidities that may elevate their risk for prolonged general anesthesia. Patients with mild obstructive symptoms may be managed electively, while patients suffering from complete gastric obstruction will require inpatient admission and urgent management. ${ }^{3-5}$ Although the stomach has a robust vascular supply, severe acute volvulus can result in gastric ischemia. Gastric strangulation is more common with severe gastric torsion (>180 degrees) and organoaxial volvulus orientation, and delay in the definitive treatment for gastric ischemia significantly increases patient mortality. Therefore, once the diagnosis of acute gastric volvulus is made, initial management should include intravenous fluid resuscitation and nasogastric tube decompression of the stomach. In select cases, upper gastrointestinal endoscopy may be useful to evaluate the

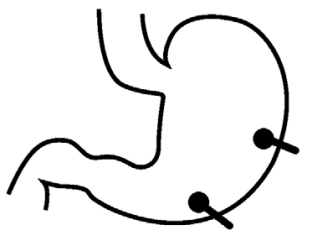

Figure 3 Gastropexy achieved by double percutaneous endoscopic gastrostomy tube placement on the body and antrum of the stomach.

presence of ischemia, to reduce the volvulus, or to aid in placement of a nasogastric tube.

The goal of definitive management of gastric volvulus is to relieve the gastric outlet obstruction and prevent recurrent volvulus. Options for management include endoscopic, laparoscopic, combined laparoendoscopic techniques, and open procedures. ${ }^{3,4,6-8}$ Manual reduction of the volvulus to relieve gastric obstruction is straightforward; however, without fixation of the stomach in its normal unobstructed orientation, recurrence of volvulus is almost guaranteed. There are two main options for prevention of recurrent volvulus: gastropexy and PEH repair.

Gastropexy is the fixation of the stomach to the diaphragm and/or anterior abdominal wall. This can be accomplished with the placement of one or two gastrostomy tubes (Figure 3), either via endoscopy or through an abdominal approach (laparoscopic or open). Even with fixation of the body of the stomach, however, the fundus may still be free enough to cause recurrent volvulus. In order to address this, laparoscopic anterior abdominal wall sutured gastropexy has also been described, which may provide a more durable fixation of the stomach without the need for placement of external tubes.

PEH repair may be more technically challenging, but offers the opportunity for definitive management of the problem, prevention of recurrence, and control of any associated reflux through concomitant fundoplication. The laparoscopic approach is associated with decreased perioperative morbidity, shorter length of stay, and lower cost. ${ }^{9}$ Furthermore, laparoscopy provides superior visualization of the esophageal hiatus and posterior mediastinum compared to open surgery. Consequently, even in the setting of an urgent operation, laparoscopic management of gastric volvulus is preferred over open techniques. ${ }^{10}$

\section{Pathogenesis}

Gastric volvulus develops due to a mobile stomach in the setting of incomplete attachment of the stomach to surrounding organs. The stomach is intrinsically a highly 
mobile organ: frequent forceful peristalsis is necessary for mechanical digestion of food. Furthermore, the size and position of the stomach can change dramatically depending on the degree to which it is filled, body habitus, and abdominal wall tone. ${ }^{11}$ Despite its highly active state, the normal stomach possesses numerous ligamentous attachments (eg, gastrosplenic, gastrophrenic, gastrohepatic, and gastroduodenal ligaments) to its surrounding organs to keep it in situ. In gastric volvulus, these normal gastric attachments are stretched and fail to maintain the stomach in its normal anatomic position. ${ }^{12}$

Primary gastric volvulus occurs with the stomach in the subdiaphragmatic position and is exclusively due to laxity in the ligamentous attachments without another contributing anatomic abnormality. This occurs in $10 \%-30 \%$ of cases of gastric volvulus. ${ }^{13}$ When primary gastric volvulus occurs, the anatomic configuration is most commonly mesenteroaxial, and generally a partial gastric outlet obstruction is present.

In the majority of gastric volvulus cases, additional anatomic abnormalities underlie the development of gastric volvulus. Previous case series have found $\mathrm{PEH}$ to be the most common condition associated with gastric volvulus. ${ }^{3-6}$ Other conditions associated with gastric volvulus include other diaphragmatic hernias (congenital or acquired), diaphragmatic paralysis, kyphoscoliosis, previous thoracic surgery, presence of intraluminal foreign objects (eg, gastric balloons, gastrostomy tubes), splenomegaly, and surgical absence of the spleen (Figure 4). ${ }^{3-6,14,15}$ Rarely, the presence of adhesions to the stomach may form an axis of rotation, setting the stage for volvulus.

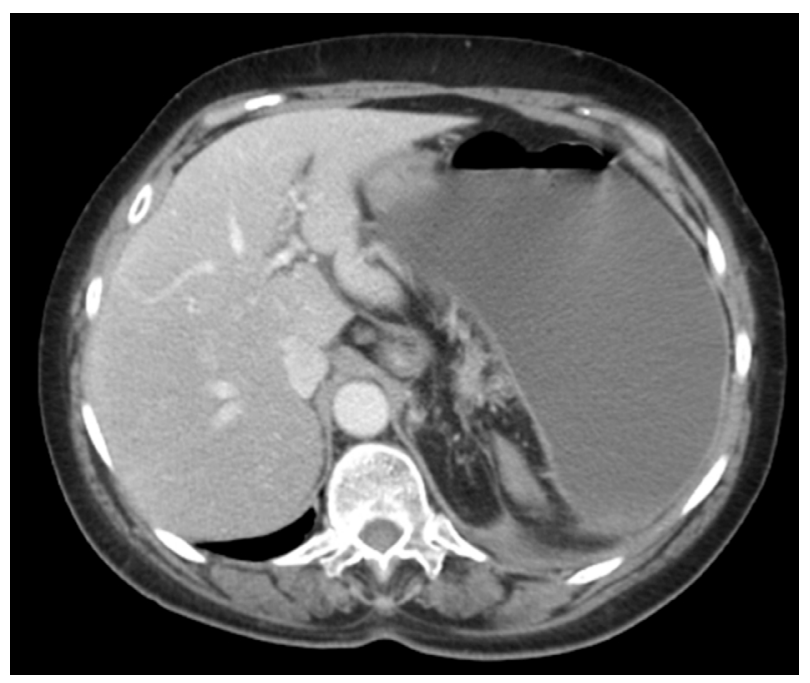

Figure 4 Computerized tomography of the abdomen demonstrating acute gastric volvulus in the setting of prior splenectomy.

Note: The absence of gastrosplenic ligament, as well as adhesive disease from prior abdominal operations, predisposes to gastric volvulus.

\section{Clinical presentation}

\section{Acute gastric volvulus}

Patients with acute gastric volvulus often present with rapid onset of upper abdominal or lower chest pain associated with symptoms of gastric outlet obstruction. In the setting of a partial obstruction, patients report nonbilious vomiting. When a complete obstruction develops, patients are unable to vomit, and dry retching results. ${ }^{14}$ In 1904, Borchardt described the triad of epigastric pain, vomiting followed by retching with inability to vomit (indicating gastroesophageal junction obstruction), and the inability to pass a nasogastric tube. ${ }^{16}$ Borchardt described this triad as being present in as many as $70 \%$ of cases. Based on the incidence of organoaxial volvulus (two-thirds of cases), this observation by Borchardt makes sense: with organoaxial volvulus, the gastroesophageal junction becomes obstructed, and passage of the nasogastric tube into the stomach is not possible. Other symptoms of acute volvulus include hematemesis due to mucosal tears following retching or underlying Cameron's ulcers in the setting of PEH, dysphagia, and dyspnea. ${ }^{3-5,17,18}$

Patients with acute gastric volvulus can be quite ill and in significant distress. Physical examination findings, however, are generally nonspecific. Vital signs may demonstrate tachycardia secondary to pain or dehydration. If the patient has aspirated, tachypnea and hypoxemia may be present. In advanced cases, gastric perforation with intra-abdominal or mediastinal contamination may result in fever. Abdominal examination may reveal generalized tenderness in the epigastrium, and acute abdominal pain with peritoneal signs can be present in the setting of gastric ischemia or necrosis and ensuing perforation. ${ }^{4,19-21}$ Acute abdominal pain may also result from solid organ complications of acute gastric volvulus, including omental avulsion, pancreatic necrosis, and disruption of splenic vessels with splenic rupture due to traction. ${ }^{14}$ Importantly, abnormal abdominal examination findings may be absent when gastric volvulus occurs in the setting of a completely intrathoracic stomach, even with advanced obstruction and gastric necrosis., ${ }^{2,5}$

\section{Chronic gastric volvulus}

Chronic gastric volvulus can refer to two separate clinical entities. First, the stomach can volvulize and maintain a partially obstructed orientation, resulting in chronic incomplete gastric outlet obstruction. These patients present with longstanding symptoms prior to seeking medical treatment. Second, over months or years, the stomach can intermittently volvulize and spontaneously return to a normal configuration 
resulting in recurrent episodes of acute obstruction. In this setting, the diagnosis of gastric volvulus can be elusive, as radiographs may not capture the stomach in an abnormal orientation. As a result, these patients may undergo multiple evaluations by their primary care physician or in an emergency department without identification of a cause of their symptoms.

Typical symptoms of chronic gastric volvulus are vague and nonspecific and may include abdominal pain, vomiting, and dysphagia. ${ }^{4}$ Additional symptoms include heartburn and regurgitation. Prior reports have pointed out the symptom overlap between chronic gastric volvulus and PEH., ${ }^{4,522}$ This should come as no surprise, given that many patients who have a PEH develop symptoms secondary to intermittent gastric volvulus.

\section{Diagnostic evaluation}

Due to the nonspecific symptoms of gastric volvulus, the diagnosis may be missed unless a high index of suspicion is maintained and appropriate diagnostic studies are obtained. However, even when a strong suspicion for gastric volvulus exists, one should consider alternative diagnoses that may present with similar symptoms, including acute myocardial infarction, pancreatitis, cholecystitis, pneumonia, and pulmonary embolism.

Diagnostic modalities used to identify gastric volvulus include chest and abdominal radiographs, upper gastrointestinal series (UGI), computerized tomography (CT), and upper gastrointestinal endoscopy.

When gastric volvulus is present, plain chest and abdominal radiographs may reveal a radiolucent hollow viscous structure, with or without an air-fluid level, in the chest (when associated with $\mathrm{PEH}$ ) or upper abdomen (Figure 5A). ${ }^{23}$ The presence of a nasogastric tube may aid in the identification of an abnormally positioned stomach (Figure 5A, arrow), and administration of contrast via the nasogastric tube may enhance this view (Figure 5B). Furthermore, chest radiographs may identify evidence of underlying anatomic abnormalities that predispose to gastric volvulus, including diaphragmatic eventration/elevated hemidiaphragm and rib fractures that suggest prior thoracoabdominal blunt force trauma. Although plain radiographs may suggest that gastric volvulus is present, additional studies are typically required to fully characterize the anatomic abnormality.

An UGI provides radiographic assessment of foregut anatomy. It allows identification of the position of the stomach, presence of a $\mathrm{PEH}$, degree of rotation of the stomach, and presence of gastric outlet obstruction (Figure 6). In
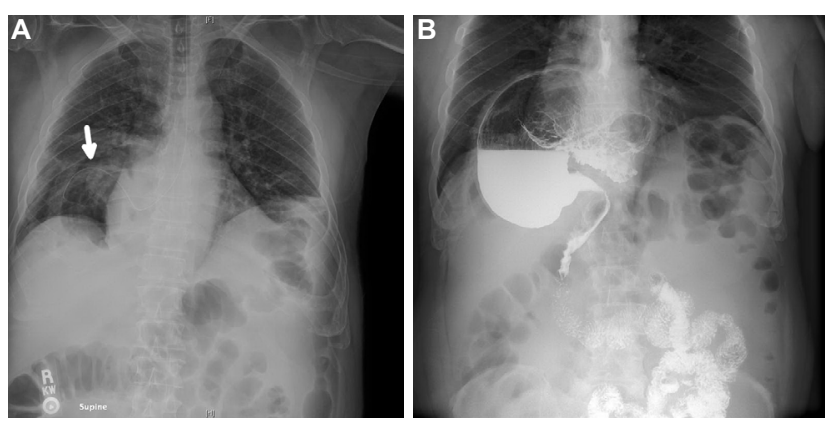

Figure 5 Organoaxial gastric volvulus.

Notes: (A) Chest radiograph demonstrating a radiolucent hollow viscous structure overlying the right hemithorax. There is a nasogastric tube (arrow) positioned in the intrathoracic stomach. (B) Administration of contrast via the nasogastric tube demonstrates a completely intrathoracic stomach with an air-fluid level. Although organoaxial volvulus and an intragastric air-fluid level is present, contrast appears to pass distally through a narrowed antrum.
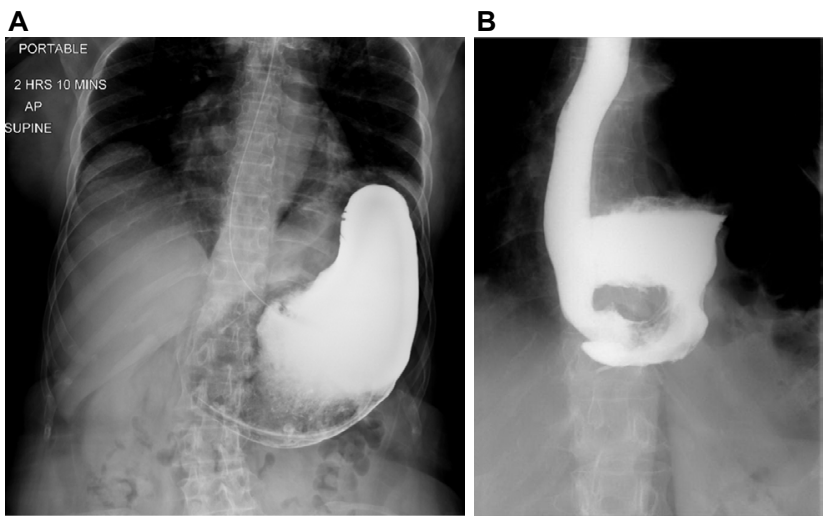

Figure 6 Upper gastrointestinal series films demonstrating complete gastric obstruction due to acute gastric volvulus involving a subdiaphragmatic stomach (A) and in the setting of paraesophageal hernia (B).

Abbreviation: AP, anteroposterior.

their series of 36 patients, Teague et al found that UGI with barium was diagnostic in $56 \%$ of patients and suggestive of gastric volvulus in $28 \%$ of patients. ${ }^{4}$ However, performing an UGI requires the presence of an on-site radiologist and thus can be inappropriate in acute gastric volvulus given the need for an expeditious diagnosis. ${ }^{3}$

CT is highly sensitive at detecting acute gastric volvulus. ${ }^{3,24}$ In patients with acute chest and abdominal pain, a CT scan is often performed and therefore may be the only test needed to recognize gastric volvulus. ${ }^{3,24}$ Furthermore, because it does not require the presence of a radiologist, it can expedite the diagnosis of acute volvulus. Features of gastric volvulus on $\mathrm{CT}$ scan are gastric distension, an antropyloric transition point (Figure 7), and position of the antrum at the same level or higher than the fundus (Figure 8). PEH and diaphragmatic hernias can also be identified. In a recent series of 36 patients, Light et al found 100\% diagnostic accuracy in 26 cases where a CT was ordered. ${ }^{3}$ It must be noted that 


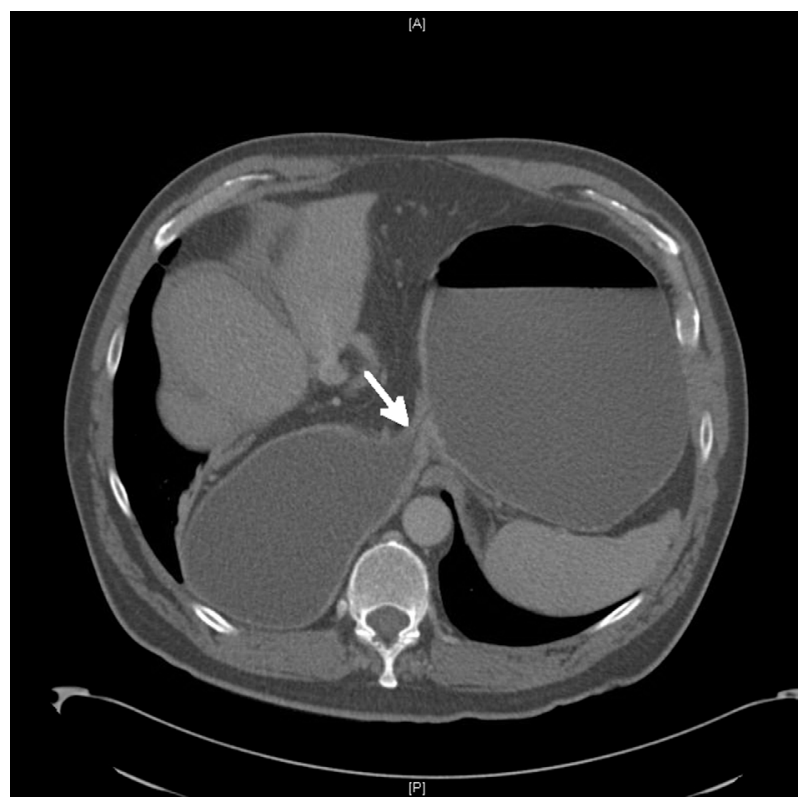

Figure 7 Computerized tomography of the abdomen demonstrating an antropyloric transition zone (arrow).

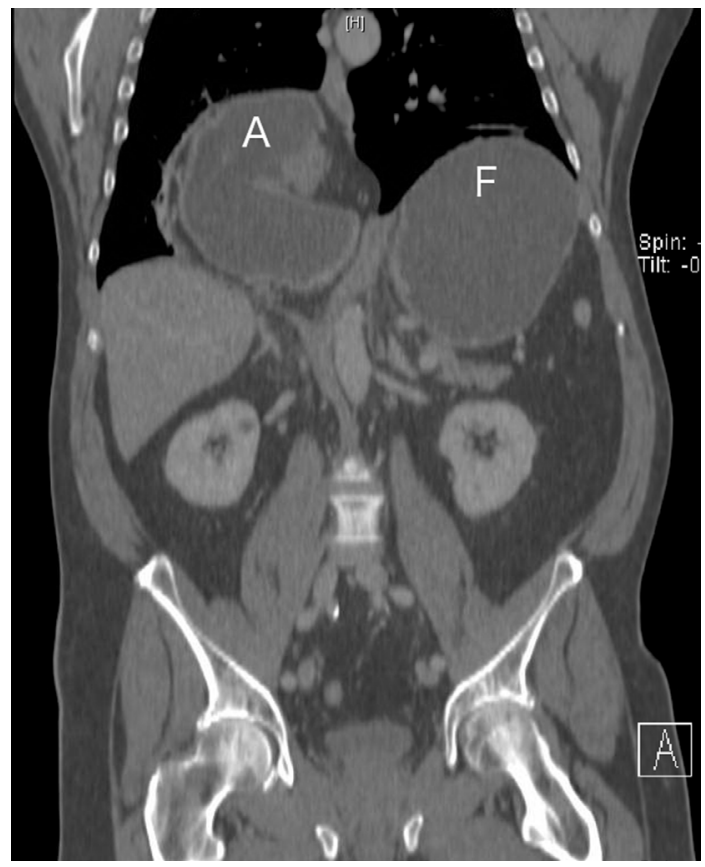

Figure 8 Coronal section of abdominal computerized tomography scan demonstrating mesenteroaxial volvulus in the setting of a large paraesophageal hernia. Notes: The gastric antrum (A) has flipped above the fundus (F) and lies in the posterior mediastinum.

while a CT scan can readily identify the presence of pneumoperitoneum, radiologic findings of gastric ischemia do not correlate well with intraoperative findings. ${ }^{23,24}$

In the setting of gastric volvulus, upper endoscopy can be both diagnostic and therapeutic (see Management section). However, in reported series, endoscopy is diagnostic of volvulus in only $28 \%-45 \%$ of patients. ${ }^{3,4}$ This is likely because endoscopy is a highly operator-dependent modality, and even skilled endoscopists may have a difficult time identifying a volvulus endoscopically. An additional explanation for this low sensitivity is that the gastric volvulus may reduce during the procedure unbeknownst to the endoscopist. Despite these shortcomings, endoscopy is the best method to assess for the presence of mucosal ischemia. ${ }^{8,25,26}$

\section{Management Overview and initial management}

A flow diagram demonstrating our management of patients with gastric volvulus is provided in Figure 9.

Chronic gastric volvulus can be managed on an elective basis according to the presence of symptoms. For patients who are symptomatic, our clinical decision-making mirrors that of patients with acute gastric volvulus without evidence of gastric ischemia (see Figure 9).

For patients who are asymptomatic and have PEH or gastric volvulus, the traditional mantra has been to expeditiously repair the abnormality regardless of the presence of symptoms to prevent future episodes of acute gastric volvulus and gastric ischemia. However, this approach has changed. In patients who are asymptomatic, and gastric volvulus is an incidental finding on a radiographic study obtained for other purposes, nonoperative management (ie, "watchful waiting") has been advocated..$^{27,28}$ This change in philosophy is based, at least in part, on the work by Stylopolous et al. ${ }^{28}$ They reported that in patients with $\mathrm{PEH}$, the risk of developing acute symptoms requiring an emergency operation is $1.16 \%$ per year. However, based on data from the National Surgical Quality Improvement Program database, emergency repair is associated with a nearly ten-fold increase in perioperative mortality compared to elective repair $(0.65 \%$ vs $5.5 \%)$. Additionally, mortality was lowest $(0.46 \%)$ in the group with elective laparoscopic repair. ${ }^{29}$ Furthermore, many patients billed as "asymptomatic" with an incidental finding of volvulus and/or PEH actually have significant symptoms on a careful history. As a result, the final decision to provide empiric operative management for incidental gastric volvulus should be based on a patient's desire to undergo repair and a balance of the risks of repair with the risks of developing acute volvulus and needing emergent repair. Unless the patient is of very advanced age or has significant medical comorbidities that place them at greatly increased risk for elective repair, we recommend operative repair to reduce the risk of subsequent acute volvulus and obstruction necessitating emergent repair. 


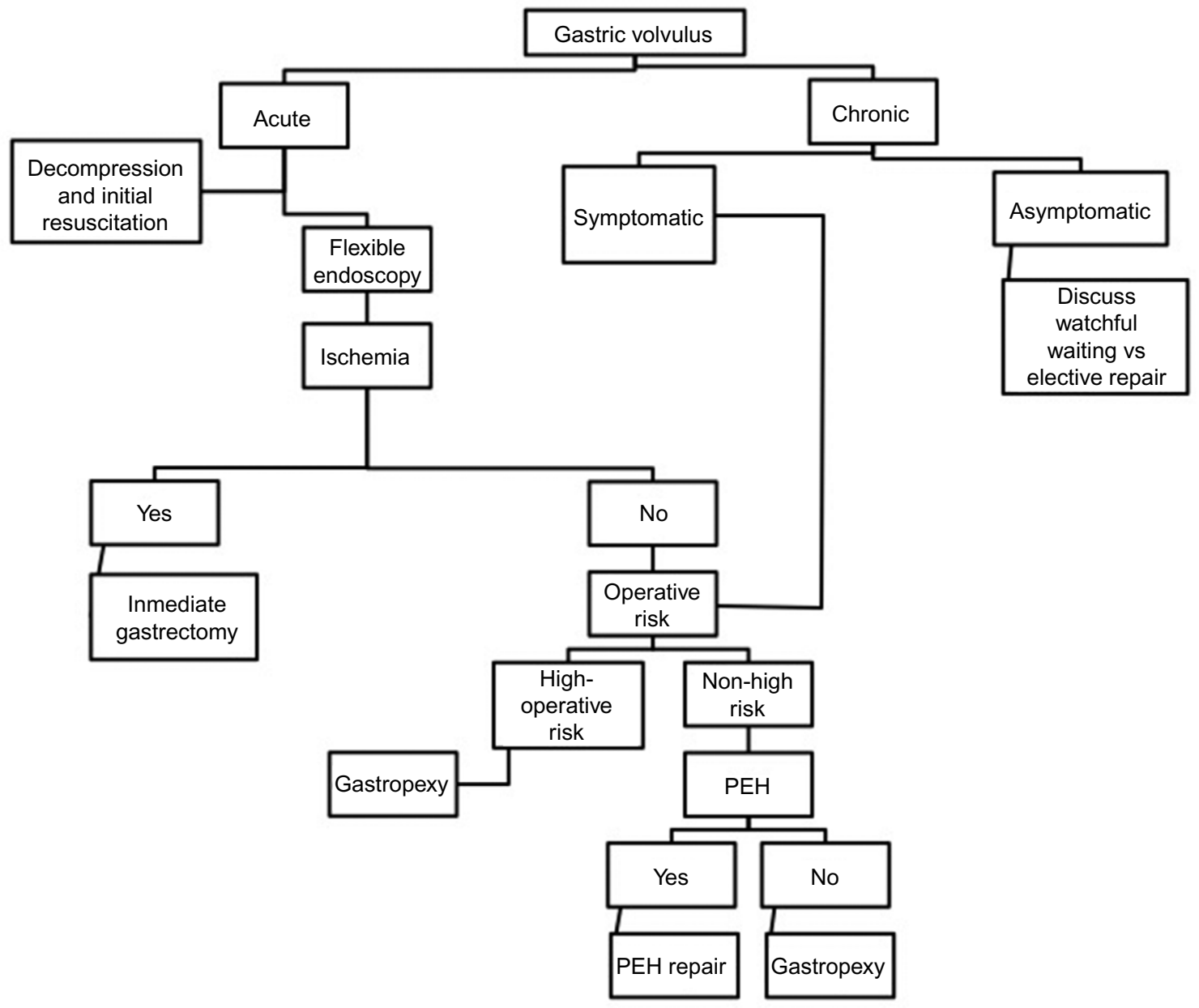

Figure 9 Management algorithm for patients with gastric volvulus.

Abbreviation: $\mathrm{PEH}$, paraesophageal hernia.

Acute gastric volvulus with high-grade gastric outlet obstruction is a surgical emergency. Initial management should include intravenous fluid resuscitation, correction of electrolyte abnormalities, and immediate gastric decompression, which can be achieved with the placement of a nasogastric tube. ${ }^{2,10}$ Importantly, even if the patient does not appear systemically ill, has significant abdominal pain, and is not tender on examination, a prompt evaluation and reduction of volvulus are necessary to prevent progression to gastric ischemia (Figure 10).

Flexible endoscopy is useful in the early management of acute gastric volvulus for several reasons. First, it can achieve gastric decompression and facilitate the placement of a nasogastric tube. This is an important benefit of endoscopy, since traditional ("blind") nasogastric tube placement may not be possible in the setting of organoaxial volvulus and an obstructed gastroesophageal junction. Second, it can restore normal anatomic configuration of the stomach and in turn relieve gastric outlet obstruction. Finally, it allows for assessment of gastric ischemia through direct visualization of the gastric mucosa. Whether by endoscopy or traditional

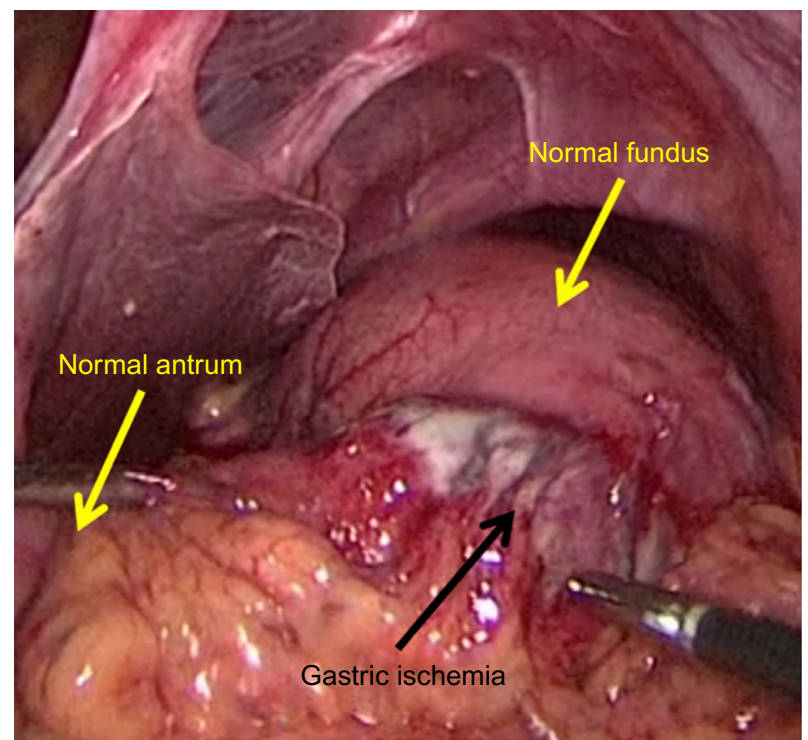

Figure 10 Intraoperative image of gastric ischemia in an elderly patient who presented with one week of gastric outlet obstruction secondary to gastric volvulus.

nasogastric tube placement, once the stomach has been decompressed, and there is no evidence of gastric ischemia, semi-elective definitive repair can be considered. Such a delay 
in definitive repair can allow for perioperative patient risk stratification and optimization in high-risk populations. ${ }^{3}$ It may also allow for temporization and transfer of the patient to a high-volume specialty center where the surgeon and perioperative resources are better equipped to manage these complex patients.

\section{Definitive treatment}

Definitive treatment of obstructed gastric volvulus depends on several important factors: 1) presence of ischemic stomach; 2) presence of a PEH; and 3) patient's perioperative risk profile. If the stomach is strangulated (Figure 10), partial or total gastrectomy is indicated. The extent of resection depends on the amount of gross necrosis or persistent ischemia after reduction of gastric volvulus. Gastric ischemia contributes significantly to patient mortality, and efforts should be made to avoid delay in performing the operation..$^{5,18}$

If the stomach is viable, then attention should be directed at reducing the volvulus and preventing recurrent volvulus. Although a wide range of procedures are available to achieve these goals, recent patient series, including our own, favor laparoscopic PEH repair or anterior abdominal wall sutured gastropexy. ${ }^{3,4,10}$

\section{Laparoscopic paraesophageal hernia repair}

Laparoscopic PEH repair in the management of gastric volvulus should follow the basic tenets of this operation. ${ }^{19-21}$ There are five steps to the standard operative repair of PEH: 1) reduction of the hernia contents into the abdomen; 2) excision of the hernia sac from the mediastinum; 3 ) mobilization of the esophagus to obtain a minimum of three centimeters of intra-abdominal esophageal length; 4) closure of the hiatus; and 5) completion of an antireflux operation. Although these operative steps are the same whether they are performed open or laparoscopically, multiple authors have reported that laparoscopic repair of hiatal hernias provides exceptional visualization, improves quality of life, and provides excellent relief from preoperative symptoms. ${ }^{9,10,20}$

Briefly, the patient is placed in a modified lithotomy or split leg position. This provides the surgeon improved ergonomics by standing between the patient's legs; the assistant stands at the patient's left. Additionally, the patient is placed in steep reverse Trendelenburg position, which allows for improved visualization of the esophageal hiatus. The patient is appropriately padded to prevent pressure ulcers and neuropathies. Preoperative antibiotics are administered to reduce the risk of surgical site infection, and subcutaneous heparin

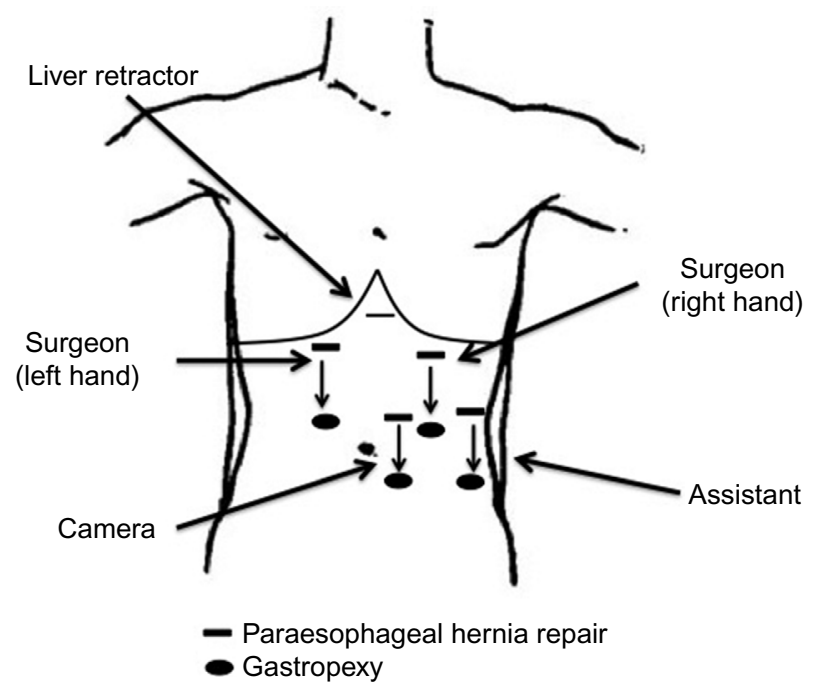

Figure II Port configuration for laparoscopic paraesophageal hernia repair (rectangles) and laparoscopic gastropexy (ovals).

Notes: When performing laparoscopic gastropexy, it is essential to shift the ports caudally on the abdominal wall to maintain visualization for gastropexy of the distal gastric body and antrum.

and sequential compression devices are used to reduce the risk of venous thromboembolic events.

Access to the abdomen is obtained using a Veress needle at Palmer's point in the left upper quadrant of the abdomen. Three additional trocars and a Nathanson liver retractor are placed (Figure 11). The table is set in a steep reverse Trendelenburg position.

The operation begins by reducing the volvulus and returning the stomach and all hernia contents to the abdominal cavity. If the stomach does not completely reduce into the abdominal cavity, no forced reduction is attempted. Beginning at a point approximately one-third to one-half along the greater curvature, the short gastric vessels are then ligated until the left crus is reached. A left crus approach is often easier in the setting of PEH, as it provides better visualization than the traditional right crus approach. It also facilitates additional reduction of the stomach as the short gastric vessels are divided. Upon reaching the left crus, the avascular fibroareolar plane between the hernia sac and medial aspect of the crus is entered. The hernia sac is dissected off the crura in a counterclockwise fashion to the top of the right crus. At this point, dissection can be continued down the right crus; however, it is often easier to start dissection at the base of the crus and then to work anteriorly, with the tissue planes meeting in the mediastinum. Once the sac is fully dissected off the crura, it can be dissected out of the mediastinum. Although the sac can be used for retraction, it is tethered to the gastroesophageal junction on the patient's left side, and too much traction can cause an esophageal tear. It is therefore 
helpful to place a Penrose drain around the esophagus as soon as it is practical to do so.

Once the sac is fully dissected and reduced, further mediastinal dissection of the esophagus is performed to achieve a minimum of $3 \mathrm{~cm}$ of intra-abdominal esophageal length. Closure of the hiatus is then performed using permanent (2-0 silk) sutures. Large hiatal hernias may prohibit simple closure. In this scenario, we perform a right-sided diaphragmatic relaxing incision along with placement of a C-shaped biologic mesh. ${ }^{30}$ The esophagus should maintain a straight orientation without angulation, and a 52 French bougie should easily pass beyond the esophageal hiatus and into the stomach. After passage of the bougie, the sac is dissected off the stomach and esophagus and resected. A lighted bougie can help with identification and preservation of the anterior vagus nerve. At this point, the fundoplication is created. If no preoperative manometry was performed, we recommend a partial (Toupet) fundoplication due to the high incidence of ineffective esophageal motility in this patient population.

It is important to recognize that laparoscopic $\mathrm{PEH}$ repair requires advanced laparoscopic skills and should only be performed by experienced gastroesophageal surgeons. In addition, laparoscopic $\mathrm{PEH}$ repair takes several hours to perform and must be completed under general anesthesia. Because gastric volvulus frequently occurs in older patients with significant comorbidities, a prolonged operation under general anesthesia may expose the patient to unacceptable perioperative risk. Furthermore, patients with acute gastric volvulus and obstruction may present to a medical center with a surgeon who is not comfortable with performing this complex repair. If the patient's condition allows for interhospital transfer, urgent referral to a tertiary medical center may be best for the patient. Alternatively, anterior abdominal wall gastropexy offers a strategy to manage patients in situations when the patient is too ill to withstand definitive repair or the required expertise is not available. ${ }^{6,31}$

\section{Anterior abdominal wall gastropexy}

Anterior abdominal wall gastropexy ("gastropexy") is the fixation of the stomach to the left hemidiaphragm and anterior abdominal wall in its normal anatomic configuration to prevent recurrent gastric volvulus. In two scenarios, gastropexy is used as an alternative to PEH repair: first, in patients who are at prohibitively high operative risk to undergo prolonged abdominal surgery; and second, when surgeons lack sufficient experience in gastroesophageal surgery to perform a complex $\mathrm{PEH}$ repair. There are three minimally invasive approaches to gastropexy - endoscopic, laparoscopic, and laparoendoscopic.
Endoscopic gastropexy is performed with the placement of one or more percutaneous endoscopic gastrostomy (PEG) tubes. This technique offers the advantages of avoiding general anesthesia and additional abdominal incisions. The first step of endoscopic gastropexy is to reduce the gastric volvulus. Typically, this can be done by advancing the scope beyond the gastroesophageal junction and insufflating the stomach with air. Even in the setting of a completely intrathoracic stomach, gastric insufflation will often reduce the stomach. ${ }^{8,31,32}$ When insufflation alone is insufficient, experienced endoscopists can perform special maneuvers to reduce the gastric volvulus. In the alpha loop technique, the endoscope is passed to the antrum, and a J-turn maneuver is performed to allow passage of the scope through the volvulus. ${ }^{8}$ The endoscope is then withdrawn back into the proximal stomach, forming an alpha loop. The tip of the endoscope is then readvanced through the narrowed lumen and torqued in an anticlockwise direction, uncoiling the alpha loop and reducing the gastric volvulus.

After reduction of the volvulus, a first PEG tube is placed on the body of the stomach along the greater curvature, and a second gastrostomy site is then selected on the gastric antrum, at least $12 \mathrm{~cm}$ from the first site (Figure 3). ${ }^{31,33} \mathrm{It}$ is important to separate these two tubes to prevent creating a fulcrum around which recurrent volvulus might occur. In addition to providing points of fixation for the stomach, PEG tubes provide enteral access for nutritional support and administration of critical enteral medications.

If the volvulus cannot be reduced by purely endoscopic means, a laparoendoscopic technique can be used. With this technique, a $5 \mathrm{~mm}$ port is placed in the umbilicus and one or two additional $5 \mathrm{~mm}$ ports are placed in the upper abdomen. These ports allow for laparoscopic reduction of the volvulus, and the PEG tubes are then placed under direct laparoscopic visualization. ${ }^{31}$ This technique is also very useful when purely endoscopic gastrostomy tube placement is contraindicatedsuch as in the setting of patient obesity that prevents adequate transabdominal illumination, when the colon is overlying the stomach, or if there is concern for gastric ischemia and diagnostic laparoscopy is needed.

In laparoscopic sutured gastropexy, the volvulus is manually reduced, and the stomach is sutured to the left hemidiaphragm and anterior abdominal wall. Most reports of this technique describe a one- or two-point sutured gastropexy with or without concomitant gastrostomy tube placement. ${ }^{25,33,34}$ However, as mentioned earlier, one or two points of fixation may be inadequate to prevent recurrent volvulus, ${ }^{35}$ and these fixation points may even provide an axis around which subsequent volvulus can occur. Consequently, 
we developed laparoscopic anterior abdominal wall gastropexy using multiple sutures along the greater curvature of the stomach to provide durable fixation and prevention of subsequent volvulus. For nearly a decade in the Center for Esophageal and Gastric Surgery at University of Washington, we have performed this technique to manage high operative risk patients with obstructive gastric volvulus

In this technique, the patient is positioned in a manner similar to PEH repair. Four ports are placed at least $5 \mathrm{~cm}$ caudal to the usual location for PEH repair, as this allows both access to the esophageal hiatus for reduction of intrathoracic stomach as well as provides space in the mid-abdomen to place multiple gastropexy sutures (Figure 11). Once the volvulus is reduced and the stomach is in its normal anatomic position, $2-0$ silk sutures are placed every $2-3 \mathrm{~cm}$ to attach the stomach to the diaphragm and anterior abdominal wall (Figure 12). The first sutures are placed high on the greater curvature and anchor the stomach to the left crus. Subsequent sutures are placed between the greater curvature and the left hemidiaphragm and finally between the antrum and the anterior abdominal wall (Figure 13).

Early in our experience, we placed a gastrostomy tube at a point along the distal body of the stomach using a Ponsky

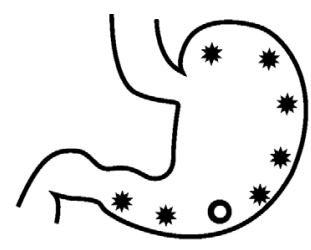

Figure 12 Schematic demonstrating placement of sutures (stars) and, when used, gastrostomy tube (circle) during anterior abdominal wall sutured gastropexy.

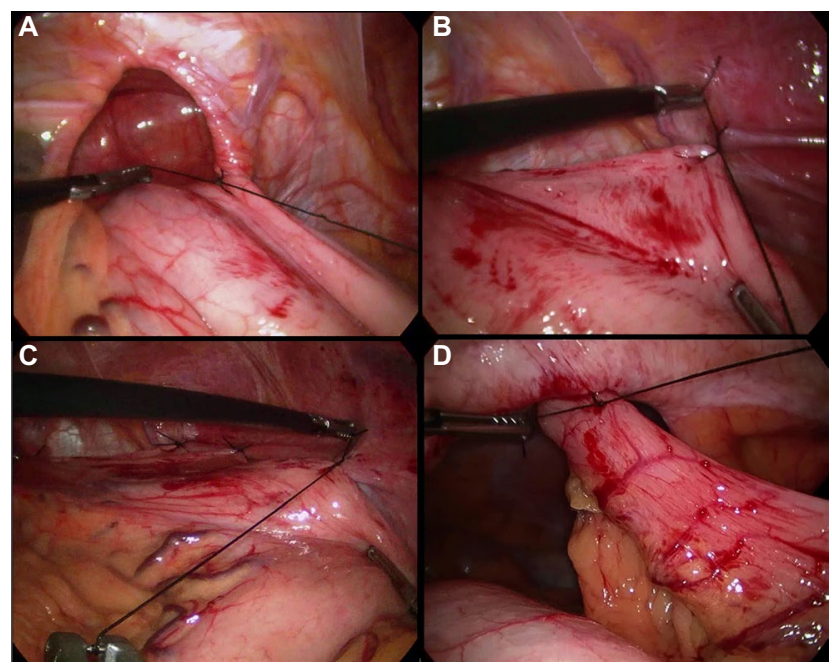

Figure 13 Intraoperative image demonstrating suture placement between the greater curve of the stomach and the left crus of the diaphragm (A), greater left hemidiaphragm (B), and anterior abdominal wall (C). The antrum is also sutured to the anterior abdominal wall (D). percutaneous endoscopy gastrostomy tube kit (Bard Access Systems; R. Bard Inc, Salt Lake City, UT, USA). This served two purposes: first, it provided an easier alternative to placement of an additional suture in an ergonomically challenging location; and second, it provided enteral access for the administration of necessary oral medication and supplemental enteral nutrition. However, in our initial series of 11 patients, 2 patients experienced premature displacement of their gastrostomy tube. Both patients required reoperation, and one died secondary to cardiac and pulmonary failure 10 days after reoperation. Furthermore, only one patient who underwent gastrostomy tube placement required the tube for enteral nutrition. Consequently, we have adopted a selective approach to placement of gastrostomy tubes in patients who are at high risk of inability to take adequate oral nutrition postoperatively (eg, severe oropharyngeal dysphagia). No recurrence of gastric volvulus was observed in a median follow-up of 3 months. ${ }^{6}$

\section{Conclusion}

Gastric volvulus remains a significant cause of gastroesophageal obstructive symptoms, and in the acute setting may be a life-threatening condition. Prompt diagnosis and management are necessary to mitigate morbidity. A high index of suspicion for gastric volvulus should be maintained in the presence of dry retching and/or vomiting, epigastric or chest pain, elderly age, and radiological signs of an intrathoracic stomach or PEH. Choice of definitive treatment of obstructed gastric volvulus depends on three major factors: 1) presence of ischemic stomach;2) presence of a $\mathrm{PEH}$; and 3) patient's perioperative risk profile. Laparoscopic PEH repair remains the mainstay for the majority of patients with acute and chronic gastric volvulus. For patients with primary volvulus and patients with significant medical comorbidities, laparoscopic anterior abdominal wall gastropexy provides a durable option and may be associated with lower perioperative morbidity.

\section{Disclosure}

The authors report no conflicts of interest in this work.

\section{References}

1. Tanner NC. Chronic and recurrent volvulus of the stomach with late results of "colonic displacement". Am J Surg. 1968;115(4):505-515.

2. Hill LD. Incarcerated paraesophageal hernia. A surgical emergency. Am J Surg. 1973;126(2):286-291.

3. Light D, Links D, Griffin M. The threatened stomach: management of the acute gastric volvulus. Surg Endosc. 2016;30(5):1847-1852.

4. Teague WJ, Ackroyd R, Watson DI, Devitt PG. Changing patterns in the management of gastric volvulus over 14 years. Br J Surg. 2000;87(3): $358-361$. 
5. Carter R, Brewer LA, Hinshaw DB. Acute gastric volvulus. A study of 25 cases. Am J Surg. 1980;140(1):99-106.

6. Yates RB, Hinojosa MW, Wright AS, Pellegrini CA, Oelschlager BK. Laparoscopic gastropexy relieves symptoms of obstructed gastric volvulus in highoperative risk patients. Am J Surg. 2015;209(5): 875-880.

7. Parker DM, Rambhajan A, Johanson K, Ibele A, Gabrielsen JD, Petrick AT. Urgent laparoscopic repair of acutely symptomatic PEH is safe and effective. Surg Endosc. 2013;27(11):4081-4086.

8. Tsang TK, Walker R, Yu DJ. Endoscopic reduction of gastric volvulus: the alpha-loop maneuver. Gastrointest Endosc. 1995;42(3):244-248.

9. Mungo B, Molena D, Stem M, Feinberg RL, Lidor AO. Thirty-day outcomes of paraesophageal hernia repair using the NSQIP database: should laparoscopy be the standard of care? J Am Coll Surg. 2014;219(2):229-236.

10. Bawahab M, Mitchell P, Church N, Debru E. Management of acute paraesophageal hernia. Surg Endosc. 2008;23(2):255-259.

11. Skandalakis JE, Colburn GL, Weidman TA, Skandalakis, P.N. Skandalakis'Surgical Anatomy. Athens, Greece: Paschalidis Medical Publications; 2004

12. Dalgaard JB. Volvulus of the stomach case report and survey. Acta Chir Scand. 1952;103(2):131-153.

13. Wasselle JA, Norman J. Acute gastric volvulus: pathogenesis, diagnosis and treatment. Am J Gastroenterol. 1993;88(10):1780-1784.

14. Rashid F, Thangarajah T, Mulvey D, Larvin M, Iftikhar SY. A review article on gastric volvulus: a challenge to diagnosis and management. Int J Surg. 2010;8(1):18-24.

15. Mercky P, Ah-Soune P, Bernardini D. A surprising complication of an intragastric balloon: a gastric volvulus. Endoscopy. 2014;46(Suppl 1):E403.

16. Borchardt M. Zun pathologie und therapie des magnevolvulus. Arch Klin Chir. 1904;74:243-260.

17. Oleynikov D, Jolley JM. Paraesophageal hernia. Surg Clin of North Am. 2015;95(3):555-565.

18. Wu M-H, Chang Y-C, Wu C-H, Kang S-C, Kuan J-T. Acute gastric volvulus: a rare but real surgical emergency. Am J Emerg Med. 2010;28(1): 118.e5-118.e7.

19. Auyang ED, Pellegrini CA. How I do it: laparoscopic paraesophageal hernia repair. J Gastrointest Surg. 2012;16(7):1406-1411.

20. Morrow EH, Oelschlager BK. Laparoscopic paraesophageal hernia repair. Surg Laparosc Endosc Percutan Tech. 2013;23(5):446-448.

21. Wolf PS, Oelschlager BK. Laparoscopic paraesophageal hernia repair. Adv Surg. 2007;41:199-210.
22. McElreath DP, Olden KW, Aduli F. Hiccups: a subtle sign in the clinical diagnosis of gastric volvulus and a review of the literature. Dig Dis Sci. 2008;53(11):3033-3036.

23. Peterson CM, Anderson JS, Hara AK, Carenza JW, Menias CO. Volvulus of the gastrointestinal tract: appearances at multimodality imaging1. Radiographics. 2009;29(5):1281-1293.

24. Millet I, Orliac C, Alili C, Guillon F, Taourel P. Computed tomography findings of acute gastric volvulus. Eur Radiol. 2014;24(12):3115-3122.

25. Eckhauser ML, Ferron JP. The use of dual percutaneous endoscopic gastrostomy (DPEG) in the management of chronic intermittent gastric volvulus. Gastrointest Endosc. 1985;31(5):340-342.

26. Kawai M, Hiramatsu M, Lee SW, et al. Endoscopy-assisted percutaneous anterior gastropexy for gastric volvulus: a minimally invasive technique using a special instrument. Endoscopy. 2013;45(Suppl 2): E151-E152.

27. Hsu Y-C, Perng CL, Chen CK, Tsai JJ, Lin HJ. Conservative management of chronic gastric volvulus: 44 cases over 5 years. World J Gastroenterol. 2010;16(33):4200-4205.

28. Stylopoulos N, Gazelle GS, Rattner DW. Paraesophageal hernias: operation or observation? Ann Surg. 2002;236(4):492-500.

29. Kaplan JA, Schecter S, Lin MY, Rogers SJ, Carter JT. Morbidity and mortality associated with elective or emergency paraesophageal hernia repair. JAMA Surg. 2015;150(11):1094-1096.

30. Crespin OM, Yates RB, Martin AV, Pellegrini CA, Oelschlager BK. The use of crural relaxing incisions with biologic mesh reinforcement during laparoscopic repair of complex hiatal hernias. Surg Endosc. 2016;30(6):2179-2185.

31. Kercher KW, Matthews BD, Ponsky JL, Goldstein SL, Yavorski RT, Sing RF, Heniford BT. Minimally invasive management of paraesophageal herniation in the high-risk surgical patient. Am J Surg. 2001; 182(5):510-514.

32. Tabo T, Hayashi H, Umeyama S, Yoshida M, Onodera H. Balloon repositioning of intrathoracic upside-down stomach and fixation by percutaneous endoscopic gastrostomy. J Am Coll Surg. 2003;197(5):868-871.

33. Tsang TK, Johnson YL, Pollack J, Gore RM. Use of single percutaneous endoscopic gastrostomy in management of gastric volvulus in three patients. Dig Dis Sci. 1998;43(12):2659-2665.

34. Ponsky J, Rosen M, Fanning A, Malm J. Anterior gastropexy may reduce the recurrence rate after laparoscopic paraesophageal hernia repair. Surg Endosc. 2003;17(7):1036-1041.

35. Toyota K, Sugawara Y, Hatano Y. Recurrent upside-down stomach after endoscopic repositioning and gastropexy treated by laparoscopic surgery. Case Rep Gastroenterol. 2014;8(1):32-38.
Open Access Surgery

\section{Publish your work in this journal}

Open Access Surgery is an international, peer-reviewed, open access journal that focuses on all aspects of surgical procedures and interventions Patient care around the peri-operative period and patient outcomes post surgery are key topics for the journal. All grades of surgery from minor cosmetic interventions to major surgical procedures are covered. Novel techniques and the

\section{Dovepress}

utilization of new instruments and materials, including implants and prostheses that optimize outcomes constitute major areas of interest. The manuscrip management system is completely online and includes a very quick and fair peer-review system, which is all easy to use. Visit http://www.dovepress.com testimonials.php to read real quotes from published authors. 